DOI: $10.1017 /$ S0963548301004989 Printed in the United Kingdom

\title{
Linear modes in the partially ionized heliosphere plasma
}

\author{
M. E. KELLUM ${ }^{1} \dagger$ and DASTGEER SHAIKH ${ }^{2} \ddagger$ \\ ${ }^{1}$ Division of Mathematics, Calhoun Community College, \\ P. O. Box 2216 Decatur, AL 35609-2216 \\ ${ }^{2}$ Department of Physics and \\ Center for Space Physics and Aeronomic Research (CSPAR), \\ University of Alabama at Huntsville, Huntsville, AL 35805. USA.
}

(Received Nov 2 2010, Accepted on Dec 4, 2010)

\begin{abstract}
The heliosphere is predominantly a partially ionized plasma that consists of electrons, ions and significant neutral atoms. Nonlinear interactions amongst these species take place through direct collision or charge exchange processes. These interactions modify linear and non linear properties of the plasma. In this work, we develop a one dimensional linear theory to investigate linear instabilities in such system. In our model, the electrons and ions are described by a single fluid compressible magnetohydrodynamic (MHD) model and are coupled self-consistently to the neutral fluid via compressible hydrodynamic equations. The coupling is mediated by the charge exchange process. Based on our self-consistent analysis, we find that the charge exchange coupling is more effective at larger length scales, and Alfvén waves are not affected by the charge exchange coupling. By contrast, the fast and slow waves are driven linearly unstable.
\end{abstract}

\section{Introduction}

Magnetohydrodynamic (MHD) plasma admits fundamental modes in the form of Alfvén, fast and slow waves. These modes are ubiquitously observed in many laboratory [5], space [6, 14] and astrophysical plasmas [1]. The Alfvén, fast and slow waves are essentially electromagnetic oscillations in a plasma that propagate along an ambient or guide magnetic field. Their propagation characteristic depends on the orientation of their wave vector relative to the velocity and magnetic field fluctuations. For instance, wave vector associated with Alfvén waves is orthogonal to the velocity field fluctuations whereas the fast and slow waves have the parallel wave vector relative to the velocity field fluctuations [14, 15].

The propagation properties of the Alfvén, fast and slow waves have long been known and well studied in the MHD plasma (in isolation). But their evolution in the presence of complex interactions or other species, such as neutral atoms/particles, is far less explored because of untractable nature of analytic as well as numerics. In the context of partially ionized (consisting of ions, electrons and significant neutrals) space and astrophysical plasmas, these waves interact with the neutral gas and govern numerous properties. Kulsrud \& Pearce (1969) in the context of cosmic

$\dagger$ mek@calhoun.edu

$\ddagger$ Email:dastgeer.shaikh@uah.edu 
ray propagation noted that the interaction of a neutral gas and plasma can damp Alfvén waves. Neutrals interacting with plasma via a relative drag process results in ambipolar diffusion [Oishi \& Mac Low 2006]. Ambipolar diffusion plays a crucial role in the dynamical evolution of the near solar atmosphere, interstellar medium, and molecular clouds and star formation. Oishi \& Mac Low (2006) investigated that the ambipolar diffusion sets a characteristic mass scale in molecular clouds. They found presence of structures below the ambipolar diffusion scale because of the propagation of compressive slow mode MHD waves at smaller scales. Leake et al (2005) showed that the lower chromosphere contains neutral atoms, the existence of which greatly increases the efficiency of wave damping due to collisional friction momentum transfer. They noted that Alfvén waves with frequencies above $0.6 \mathrm{~Hz}$ are completely damped and frequencies below $0.01 \mathrm{~Hz}$ are unaffected. They undertook a quantitative comparative study of the efficiency of the role of (ion-neutral) collisional friction, viscous and thermal conductivity mechanisms in damping MHD waves in different parts of the solar atmosphere. It was pointed out by the authors that a correct description of MHD wave damping requires the consideration of all energy dissipation mechanisms through the inclusion of the appropriate terms in the generalized Ohms law, the momentum, energy and induction equations. Padoan et al (2000) calculated frictional heating by ion-neutral (or ambipolar) drift in turbulent magnetized molecular clouds and showed that the ambipolar heating rate per unit volume depends on field strength for constant rms Mach number of the flow, and on the Alfvénic Mach number.

In the context of molecular clouds, the role of ion-neutral collision has been investigated by Balsara (1996). It was found that momentum of the plasma is governed predominantly by the slow comoving neutrals that tend to dissipate Cloud's magnetic field. Balsara (1996) described a linear analysis by considering neutral momentum in the presence of gravitational field of the Clouds that dominates the plasma momenta because of their higher masses. Hence they ignored the inertial terms in plasma momentum equation. Balsara (1996) reported that in super Alfvénic regime, the slow waves propagate without significant damping near short wavelengths, while the fast and Alfvén waves undergo rapid damping.

In addition to the neutral gas interacting collisionally with the plasma, it undergoes charge exchange process in the heliosphere and in the local interstellar medium (ISM) $[17,12]$. Various regions of interactions in the heliosphere are depicted in Fig (1). The charge exchange process knocks off electron from a neutral and makes it a ion. This electron is captured by the plasma ion which then turns into a neutral atom. This process conserves the density and but not momentum and energy of the plasma protons and $\mathrm{H}$ neutral gas. In the local interstellar medium (LISM), just beyond the heliosphere, the low density plasma and neutral hydrogen $(\mathrm{H})$ gas are coupled primarily through the process of charge exchange. Thus on sufficiently large temporal and spatial scales, a partially ionized plasma is typically regarded as equilibrated. Nonetheless it remains to be seen as to what happens to these MHD modes in the presence of charge exchange process, both in the inner and outer helioshpere regions. We address this question in this paper based on a linear theory that describes the coupled plasma-neutral system mediated by the charge exchange process within the realm of heliosphere. While obviously of interest to the solar wind-LISM interaction $[17,12]$, this work will have interesting implications for any shock, structures, or turbulent fluctuations embedded in a partially ionized medium, ranging from supernova shocks (SNR) to cometary bow shocks. 


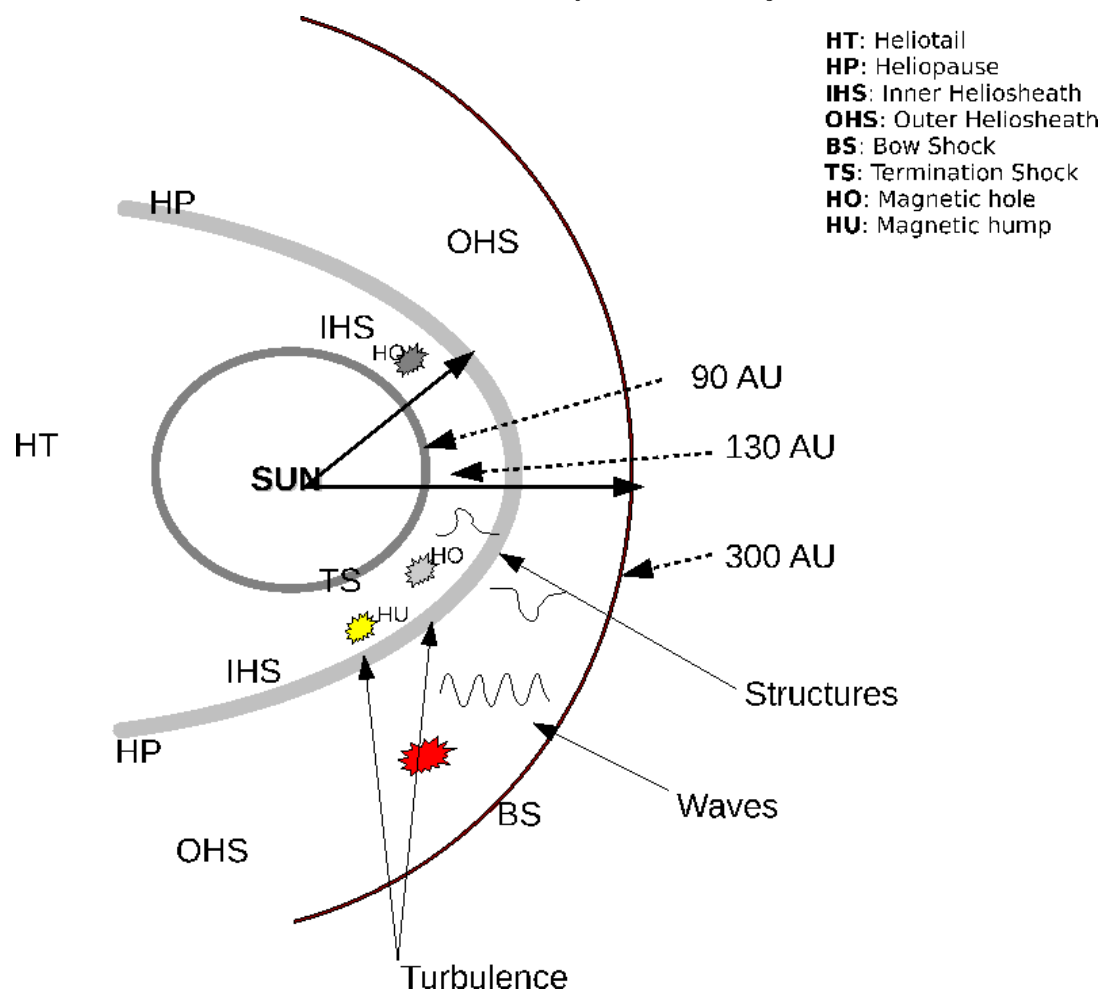

Figure 1. Schematic overview of different regions in the global heliosphere. The solar wind emanating from the Sun propagates outward and interacts with partially ionized interstellar gas predominantly via charge exchange, and creates pick up ions (PUIs). At the termination shock (TS), the supersonic SW decelerated, heated, compressed becoming subsonic, in the heliosheath, and again interacts with interstellar neutrals via charge exchange before it reaches heliopause (HP). The subsonic SW flows down into the heliotail. During its journey from the Sun to the HP, the solar wind plasma develops multitude of length and time scales that interact with the partially ionized interstellar gas, TS, and nonlinear structures develop in a complex manner.

In section 2, we describe the governing equations of the coupled plasma-neutral system in the heliosphere plasma. Importance of the charge exchange is discussed. Section 3 deals with the linear theory of MHD modes in the presence of charge exchange. Here the plasma and neutral fluids are coupled via charge exchange. Because of the analytic complexities, we focus only on one dimensional calculation. The solution of linear dispersion relation for the coupled plasma-neutral system is described in section 4 . In section 5 , we summarize our results.

\section{Model equations}

Within the paradigm of the heliosphere plasma, the plasma and neutral fluid remain close to thermal equilibirium and behave as Maxwellian fluids. They are coupled through charge exchange forces. There may additionally be direct collision between the two components. We however ignore it for our current calculation. In general, the fluid model describing nonlinear turbulent processes, in the presence of charge exchange and collision, can be described in terms of the plasma density $(\rho)$, velocity 
$(\mathbf{U})$, magnetic field $(\mathbf{B})$, pressure $(P)$ components according to the conservative form

where,

$$
\frac{\partial \mathbf{F}_{p}}{\partial t}+\nabla \cdot \mathbf{Q}_{p}=\mathcal{Q}_{p, n},
$$

and

$$
\begin{gathered}
\mathbf{F}_{p}=\left[\begin{array}{c}
\rho \\
\rho \mathbf{U} \\
\mathbf{B} \\
e_{p}
\end{array}\right], \mathbf{Q}_{p}=\left[\begin{array}{c}
\rho \mathbf{U} \\
\rho \mathbf{U U}+\frac{P}{\gamma-1}+\frac{B^{2}}{8 \pi}-\mathbf{B B} \\
\mathbf{U B}-\mathbf{B U} \\
e_{p} \mathbf{U}-\mathbf{B}(\mathbf{U} \cdot \mathbf{B})
\end{array}\right], \\
0 \\
\mathcal{Q}_{p, n}=\left[\begin{array}{c}
\mathbf{Q}_{M, p, n}+\mathbf{F}_{p, n} \\
0 \\
Q_{E, p, n}+\mathbf{U} \cdot \mathbf{F}_{p, n}
\end{array}\right]
\end{gathered}
$$

$$
e_{p}=\frac{1}{2} \rho U^{2}+\frac{P}{\gamma-1}+\frac{B^{2}}{8 \pi} .
$$

Note the presence of the source terms $Q$ that couple the plasma self-consistently to the neutral gas. The above set of plasma equations is supplimented by $\nabla \cdot \mathbf{B}=0$ and is coupled self-consistently to the neutral density $(N)$, velocity $(\mathbf{V})$ and pressure $\left(P_{n}\right)$ through a set of hydrodynamic fluid equations,

$$
\frac{\partial \mathbf{F}_{n}}{\partial t}+\nabla \cdot \mathbf{Q}_{n}=\mathcal{Q}_{n, p},
$$

where,

$$
\begin{gathered}
\mathbf{F}_{n}=\left[\begin{array}{c}
N \\
N \mathbf{V} \\
e_{n}
\end{array}\right], \mathbf{Q}_{n}=\left[\begin{array}{c}
N \mathbf{V} \\
N \mathbf{V} \mathbf{V}+\frac{P_{n}}{\gamma-1} \\
e_{n} \mathbf{V}
\end{array}\right], \\
\mathcal{Q}_{n, p}=\left[\begin{array}{c}
0 \\
\mathbf{Q}_{M, n, p}+\mathbf{F}_{n, p} \\
Q_{E, n, p}+\mathbf{V} \cdot \mathbf{F}_{n, p}
\end{array}\right] \\
e_{n}=\frac{1}{2} N V^{2}+\frac{P_{n}}{\gamma-1} .
\end{gathered}
$$

Equations (2.1) to (2.2) form an entirely self-consistent description of the coupled plasma-neutral turbulent fluid in a partially ionized medium.

Several points are worth noting. The charge-exchange momentum sources in the plasma and the neutral fluids, i.e. Eqs. (2.1) \& (2.2), are described respectively by terms $\mathbf{Q}_{M, p, n}\left(\mathbf{U}, \mathbf{V}, \rho, N, T_{n}, T_{p}\right)$ and $\mathbf{Q}_{M, n, p}\left(\mathbf{V}, \mathbf{U}, \rho, N, T_{n}, T_{p}\right)$. These expressions are described in Pauls et al (1995) and Shaikh \& Zank (2008, 2010). A swapping of the plasma and the neutral fluid velocities in this representation corresponds, for instance, to momentum changes (i.e. gain or loss) in the plasma fluid as a result of charge exchange with the neutral atoms (i.e. $\mathbf{Q}_{M, p, n}\left(\mathbf{U}, \mathbf{V}, \rho, N, T_{n}, T_{p}\right)$ in Eq. (2.1)). Similarly, momentum change in the neutral fluid by virtue of charge exchange with the plasma ions is described by $\mathbf{Q}_{M, n, p}\left(\mathbf{V}, \mathbf{U}, \rho, N, T_{n}, T_{p}\right)$ in Eq. (2.2).

The underlying coupled fluid model can be non-dimensionalized straightforwardly using a typical scale-length $\left(\ell_{0}\right)$, density $\left(\rho_{0}\right)$ and velocity $\left(v_{0}\right)$. The normalized 

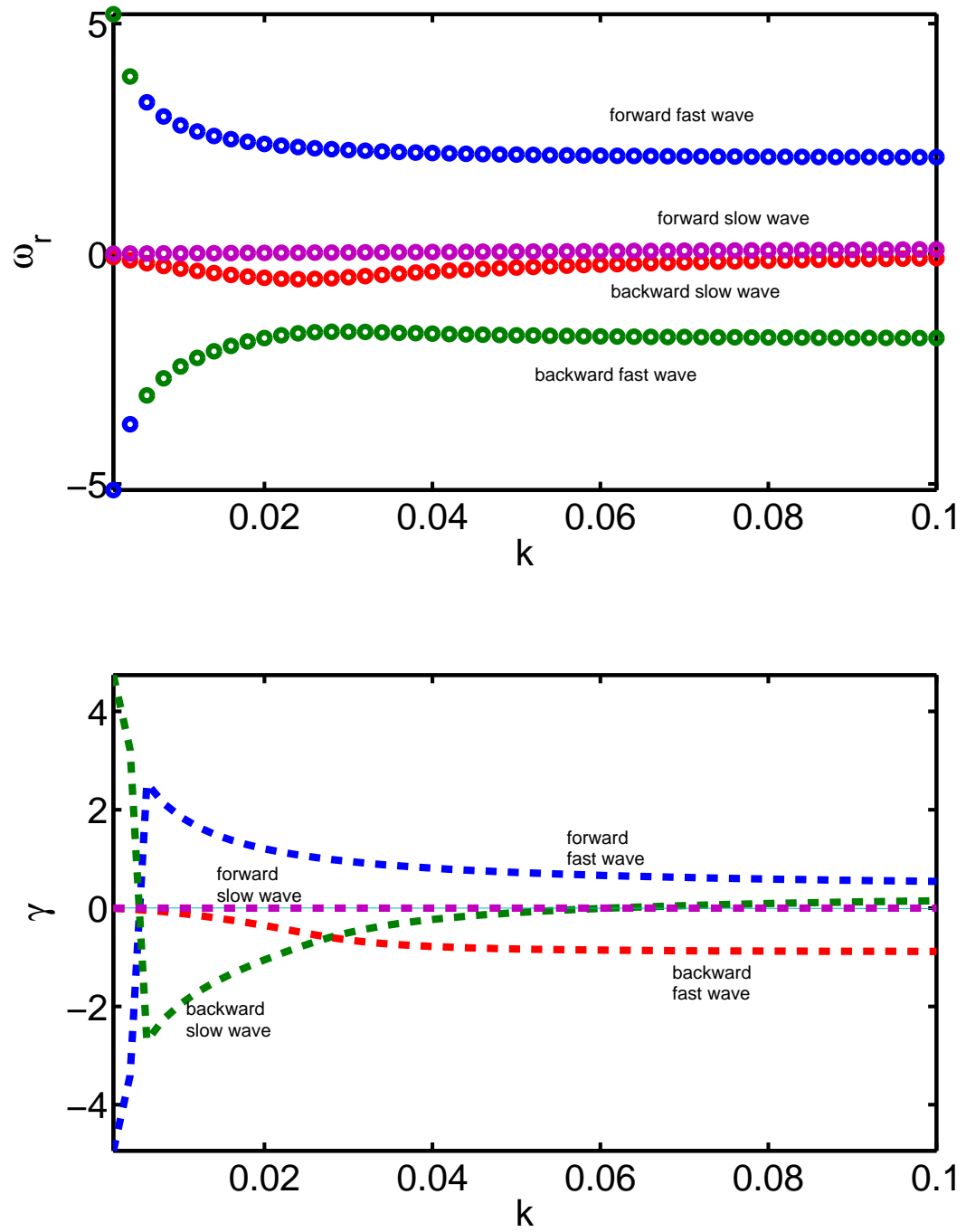

Figure 2. Solution of linear dispersion relation in the smaller $k$ (large scale) regime characterizes different MHD and hydrodynamic modes in the coupled plasma-neutral system. Upper panel describes real part of the frequency where forward/backward fast and slow waves are shown. Growth and damping of linear instability are shown in the lower panel, where $\gamma=0$ root corresponds to the sound wave. The latter is stable at lower $k$ 's.

plasma density, velocity, energy and the magnetic field are respectively; $\bar{\rho}=\rho / \rho_{0}, \overline{\mathbf{U}}=$ $\mathbf{U} / v_{0}, \bar{P}_{p}=P_{p} / \rho_{0} v_{0}^{2}, \overline{\mathbf{B}}=\mathbf{B} / v_{0} \sqrt{\rho_{0}}$. The corresponding neutral fluid quantities are $\bar{N}=N / \rho_{0}, \overline{\mathbf{V}}=\mathbf{V} / v_{0}, \bar{P}_{n}=P_{n} / \rho_{0} v_{0}^{2}$. The momentum and the energy chargeexchange terms, in the normalized form, are respectively $\overline{\mathbf{Q}}_{m}=\mathbf{Q}_{m} \ell_{0} / \rho_{0} v_{0}^{2}, \bar{Q}_{e}=$ $Q_{e} \ell_{0} / \rho_{0} v_{0}^{3}$. The non-dimensional temporal and spatial length-scales are $\bar{t}=t v_{0} / \ell_{0}, \overline{\mathbf{x}}=$ $\mathbf{x} / \ell_{0}$. Note that we have removed bars from the set of normalized coupled model equations (2.1) \& (2.2). 


\section{Linear theory}

To avoid mathematical complications associated with the coupled plasma neutral system, we develop a one dimensional linear theory to investigate growth/damping of fundamental MHD modes in the heliosphere plasma. One dimensional theory offers not only a better insight into the complex (charge exchange) interaction processes, but it also is easier (compared to two dimensional treatment) to deal with analytically. Furthermore, it describes dominant features of the characteristic modes of the coupled plasma-neutral system. In our linear theory, the charge exchange term $Q_{m}$ in the momentum equation is proportional to $\nu \rho(U-V)$ [Florinski et al 2005] where $\nu$ is the charge exchange frequency. This is a simpler form of the charge exchange force. For more complicated structure of the charge exchange force, we employ higher order terms for the nonlinear simulations. This is described in Shaikh \& Zank (2010) where nonlinear stage of mode coupling instability in two dimension was investigated. Our results, to be deduced below, help explain some of the features of the nonlinear simulations described in Shaikh \& Zank (2010). The one-dimensional plasma equations in the normalized form are described as;

$$
\begin{gathered}
\frac{\partial \rho}{\partial t}+\frac{\partial}{\partial x}(\rho U)=0 \\
\rho \frac{\partial U}{\partial t}+\rho U \frac{\partial U}{\partial x}+\frac{\partial P}{\partial x}=-\nu \rho(U-V), \\
\frac{\partial P}{\partial t}+U \frac{\partial P}{\partial x}+\gamma P \frac{\partial U}{\partial x}=\nu \rho\left[\frac{\gamma-1}{2}(U-V)^{2}+v_{t h}^{2}-\frac{P}{2 \rho}\right], \\
\frac{\partial B}{\partial t}=B \frac{\partial U}{\partial x}-U \frac{\partial B}{\partial x}-B \frac{\partial U}{\partial x} .
\end{gathered}
$$

Here $\nu=\sigma U^{*} N$ is charge exchange frequency that depends on the charge exchange cross section $(\sigma)$ and the relative difference in the speed of neutral and plasma components. The plasma thermal speed is $v_{t h}=\sqrt{k T_{i} / m_{i}}$ and $\gamma$ is the specific heat ratio (it is $5 / 3$ in our model). Note that the sign of the momentum charge exchange force is negative. This means that charge exchange process tends to decrease plasma momentum relative to the neutral fluid. The magnetic field is unaffected by the charge exchange process and it continues to be frozen into the plasma component of the flow. Similar equations for the neutral fluid density $(N)$, velocity $(V)$ and pressure $\left(P_{n}\right)$ can be described as follows.

$$
\begin{gathered}
\frac{\partial N}{\partial t}+\frac{\partial}{\partial x}(N V)=0 \\
N \frac{\partial V}{\partial t}+N V \frac{\partial V}{\partial x}+\frac{\partial P_{n}}{\partial x}=+\nu \rho(U-V) \\
\frac{\partial P_{n}}{\partial t}+V \frac{\partial P_{n}}{\partial x}+\gamma P_{n} \frac{\partial V}{\partial x}=\nu \rho\left[\frac{\gamma-1}{2}(U-V)^{2}+v_{t h}^{2}-\frac{P_{n}}{2 N}\right] .
\end{gathered}
$$

Unlike plasma, the momentum charge exchange force corresponding to the neutral fluid is positive. This leads to an enhancement in the neutral momentum relative to the plasma fluid. The rate of decay or enhancement of both the plasma and neutral momentum also depends on the relative speed between the two species. It is evident from Eqs. (3.1) to (3.7) that the plasma and neutral fluids are coupled through charge exchange sources. 

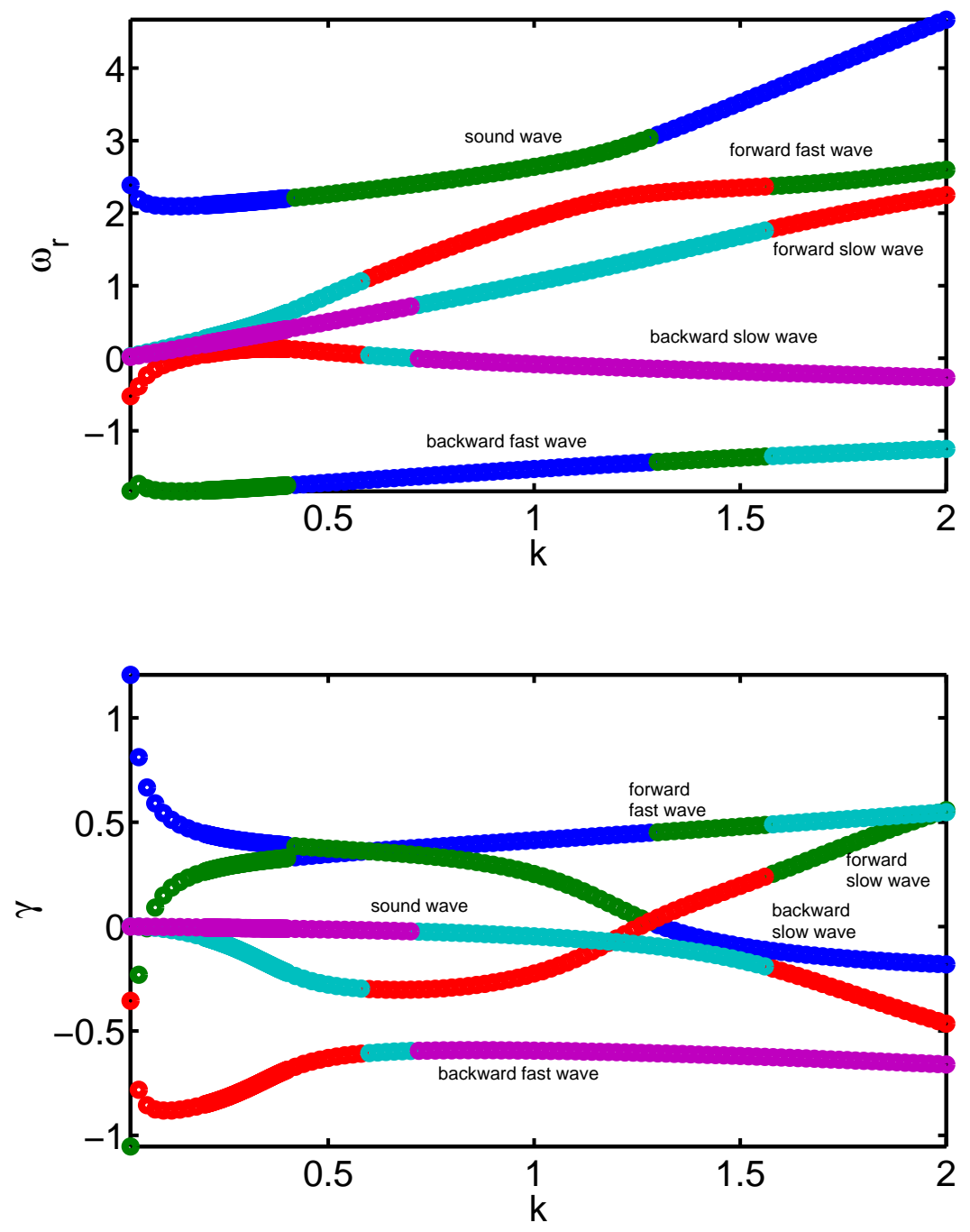

Figure 3. Linear instability in the moderately higher $k$ regime. Backward and forward fast/slow modes are shown respectively by the lower and upper curves in the upper panel. Interestingly the modes that decay in the small $k$ regime, tend to grow eventually when $k$ is order unity. This is shown in the lower panel.

We next carry out a linear analysis by assuming fixed (or constant) background quantities around which the small amplitude perturbed quantities are expanded. The amplitude of the linear perturbation is assumed to be smaller compared to the background quantity for a valid description of the linear theory. All the perturbed variables are expressed in the form of waves with frequency $\omega$ and wavenumber $k$. The plasma quantities are described as

$$
\begin{gathered}
\rho(x, t)=\rho_{0}+\tilde{\rho}_{k} e^{i k x-i \omega t}, \\
U(x, t)=U_{0}+\tilde{U}_{k} e^{i k x-i \omega t}, \\
P(x, t)=P_{0}+\tilde{P}_{k} e^{i k x-i \omega t},
\end{gathered}
$$




$$
B(x, t)=B_{0}+\tilde{B}_{k} e^{i k x-i \omega t},
$$

where $\tilde{\rho}_{k}(x, t), \tilde{U}_{k}(x, t) \tilde{P}_{k}(x, t)$ and $\tilde{B}_{k}(x, t)$ are respectively small amplitude density, velocity, pressure and magnetic field fluctuations. Note that the linear frequency $\omega$ is essentially a complex quantity i.e. $\omega=\omega_{r}+i \gamma$ where the linear part corresponds to the wave. By contrast the imaginary part describes growth (if positive) or damping (if negative) associated with the linear perturbations. We are interested in determining $\gamma(k)$ in our work.

The corresponding linear expansion for the neutral fluid is

$$
\begin{gathered}
N(x, t)=N_{0}+\tilde{N}_{k} e^{i k x-i \omega t}, \\
V(x, t)=V_{0}+\tilde{V}_{k} e^{i k x-i \omega t}, \\
P_{n}(x, t)=P_{n_{0}}+\tilde{P}_{n k} e^{i k x-i \omega t},
\end{gathered}
$$

where $N_{0}, V_{0}, P_{n_{0}}$ are respectively the background constant neutral density, velocity and pressure, and $\tilde{N}_{k}, \tilde{V}_{k}, \tilde{P}_{n k}$ are the amplitude of corresponding perturbed variables.

On linearizing Eqs. (3.1) to (3.7), we obtain

$$
\begin{gathered}
-i \omega \tilde{\rho}_{k}+i k\left(\rho_{0} \tilde{U}_{k}+U_{0} \tilde{\rho}_{k}\right)=0, \\
-i \omega \rho_{0} \tilde{U}_{k}+\rho_{0} U_{0} i k \tilde{U}_{k}+i k P_{k}=-\nu\left(\rho_{0} \tilde{U}_{k}+\tilde{\rho}_{k} U_{0}-\rho_{0} \tilde{V}_{k}-\tilde{\rho}_{k} V_{0}\right), \\
-i \omega \tilde{P}_{k}+i k U_{0} \tilde{P}_{k}+i k P_{0} \gamma \tilde{U}_{k}=\nu \tilde{\rho}_{k}\left[\frac{\gamma-1}{2}\left(U_{0}-V_{0}\right)^{2}+v_{t h}^{2}-\frac{P_{0}}{2 \rho_{0}}\right], \\
\omega=k U_{0}, \\
-i \omega \tilde{N}_{k}+i k\left(N_{0} \tilde{V}_{k}+V_{0} \tilde{N}_{k}\right)=0, \\
-i \omega N_{0} \tilde{V}_{k}+N_{0} V_{0} i k \tilde{V}_{k}+i k P_{n_{k}}=\nu\left(\rho_{0} \tilde{U}_{k}+\tilde{\rho}_{k} U_{0}-\rho_{0} \tilde{V}_{k}-\tilde{\rho}_{k} V_{0}\right), \\
-i \omega \tilde{P}_{n_{k}}+i k V_{0} \tilde{P}_{n_{k}}+i k P_{n_{0}} \gamma \tilde{V}_{k}=\nu \tilde{\rho}_{k}\left[\frac{\gamma-1}{2}\left(U_{0}-V_{0}\right)^{2}+v_{t h}^{2}-\frac{P_{n_{0}}}{2 N_{0}}\right] .
\end{gathered}
$$

It is noted that the Alfvén wave frequency, $\omega=k U_{0}$, is decoupled trivially from the neutral fluid despite charge exchange coupling interactions. This indicates that Alfvén waves are not affected by the charge exchange interactions. This result can be elucidated as follows. Firstly, the charge exchange process tends to swap the plasma ions with the neutral particles. During this process, the wave activity is not tampered by the charge exchange. Secondly, the magnetic field is unperturbed by the charge exchange process and it continues to remain frozen in the plasma component of the flow. Hence the linear frequency of Alfvén waves continues to remain unchanged. However, this scenario can change during the nonlinear evolution in that Alfvén waves are shown to exhibit modulation [Shaikh \& Zank 2010] mitigated by rapid nonlinear growth and damping that occur alternatively in the coupled plasma-neutral system.

After eliminating all the perturbed variables, we get the fifth order linear dispersion relation with complex coefficients,

$$
A_{5} \omega^{5}+A_{4} \omega^{4}+A_{3} \omega^{3}+A_{2} \omega^{2}+A_{1} \omega+A_{0}=0 .
$$

Note further that the sixth order term in the above polynomial exists separately in the form of the Alfvénic branch (i.e. $\omega=k U_{0}$ ) which is shown to decouple 
because the magnetic field, critical in exciting Alfvén waves, is unaffected largely by the charge exchange process. The magnetic field fluctuations are embeded into the plasma component and are affected only indirectly by the plasma momentum flows. Nonetheless, the complex coefficients in Eq. (3.15) are given below;

$$
\begin{aligned}
& A_{5}=N_{0}, \\
& A_{4}=i \rho_{0}\left(N_{0} \nu+\nu\right)-2 V_{0} N_{0} k-\rho_{0} U_{0} N_{0} k-2 U_{0} N_{0} k, \\
& A_{3}=i \rho_{0} U_{0}\left(-N_{0} k \nu-2 k \nu\right)+i \rho_{0} V_{0}\left(-3 N_{0} k \nu-k \nu\right) \\
& +i \rho_{0}^{2} U_{0} k \nu+\gamma\left(-N_{0} P_{0} k^{2}-P_{n_{0}}\right)+\rho_{0}\left(2 U_{0} V_{0} N_{0} k\right. \\
& \left.+2 U_{0}^{2} N_{0} k^{2}\right)+V_{0}^{2} N_{0} k^{2}+4 U_{0} V_{0} N_{0} k^{2}+U_{0}^{2} V_{0} N_{0} k^{2}, \\
& A_{2}=\rho_{0} N_{0} k^{2} \nu \delta_{p}-\rho U_{0} V_{0}^{2} N_{0} k^{3}-4 U_{0} V_{0} N_{0} k^{3}-U_{0}^{3} N_{0} k^{3} \\
& +i \rho_{0} U_{0} V_{0}\left(3 N_{0} k^{2} \nu+2 k^{2} \nu\right)+i \rho_{0} 3 V_{0}^{2} N_{0} k^{2} \nu+ \\
& \left.i \rho_{0}^{2}\left(U_{0} V_{0} k^{2} \nu-2 U_{0}^{2} k^{2} \nu\right)+i \rho_{0}^{\nu} U_{0}^{2} k^{2}\right) \\
& +\gamma i\left(-\rho_{0} P_{0} k^{2} \nu-P_{n_{0}} \rho_{0}\right)+2 \gamma V_{0} N_{0} P_{0} k^{3} \\
& +\gamma U_{0} N_{0} P_{0} k^{3}+P_{n_{0}}\left(\rho_{0} U_{0} k+2 U_{0} k\right) \gamma-2 U_{0} V_{0}^{2} N_{0} k^{3}-2 U_{0}^{2} V_{0} N_{0} k^{3} \text {, } \\
& A_{1}=i \rho_{0}\left(-2 V_{0} N_{0} k^{3} \nu \delta_{p}-V_{0}^{3} N_{0} k^{3} \nu-3 U_{0} V_{0}^{2} N_{0} k^{3} \nu-\right. \\
& \left.U_{0}^{2} V_{0} k^{2} \nu\right)+i \rho_{0}^{2}\left(U_{0}^{3} k^{3} \nu-2 U_{0}^{2} V_{0} k^{3} \nu\right)+\rho_{0}^{2}\left(\nu^{2} \delta_{p}\right. \\
& \left.-k^{2} \nu^{2} \delta_{p}\right)+\gamma i \rho_{0}\left(V_{0} P_{0} k^{3} \nu+U_{0} P_{0} k^{3} \nu\right) \\
& +i P_{n_{0}} \rho_{0}\left(2 U_{0} k-\nu U_{0} k+V_{0} k\right)-i V_{0}^{2} N_{0} P_{0} k^{4}-2 i U_{0} V_{0} N_{0} P_{0} k^{4} \\
& +i P_{n_{0}}\left(-2 \rho_{0} U_{0}^{2} k^{2}-U_{0}^{2} k^{2}\right)+\rho_{0}\left(2 U_{0}^{3} V_{0} N_{0} k^{4}+2 U_{0}^{2} V_{0}^{2} N_{0}\right) \\
& +U_{0}^{2} V_{0}^{2} N_{0} k^{4}+\gamma P_{n_{0}} P_{0} \\
& A_{0}=-\rho_{0} U_{0}^{3} V_{0} N_{0} k^{5}-\gamma^{2} P_{n_{0}} U_{0} P_{0} k \\
& +\gamma i P_{n_{0}} \rho_{0}\left[-k^{2} \nu \delta_{p}+U_{0}^{2} k^{2}(\nu-1)-U_{0} V_{0} k^{2}\right] \\
& -i \rho_{0} U_{0} V_{0} P_{0} \nu k^{4}+\gamma U_{0} V_{0}^{2} N_{0} P_{0} k^{5}+P_{n_{0}} \rho_{0} U_{0}^{3} k^{3} \\
& +i \rho_{0} V_{0}^{2} k^{4} \nu \delta_{p}+i \rho_{0} U_{0} V_{0}^{3} N_{0} k^{4} \nu+i \rho_{0} U_{0}^{3} V_{0} k^{4} \nu \\
& +\rho_{0}^{2}\left(V_{0} k^{3} \nu^{2} \delta_{p}-U_{0} k \delta_{n} \nu^{2}\right), \\
& \delta_{p}=\left[\frac{\gamma-1}{2}\left(U_{0}-V_{0}\right)^{2}+v_{t h}^{2}-\frac{P_{0}}{2 \rho_{0}}\right], \\
& \delta_{n}=\left[\frac{\gamma-1}{2}\left(U_{0}-V_{0}\right)^{2}+v_{t h}^{2}-\frac{P_{n_{0}}}{2 N_{0}}\right] \text {. }
\end{aligned}
$$

\section{Solution of the dispersion relation}

We next describe the solution of linear dispersion relation, Eq. (3.15), for the coupled plasma-neutral system in the heliosphere. To access a broader spectrum, we solve Eq. (3.15) in three distinct regime, namely the short length scale $\left(k / k_{c}>1\right)$, 

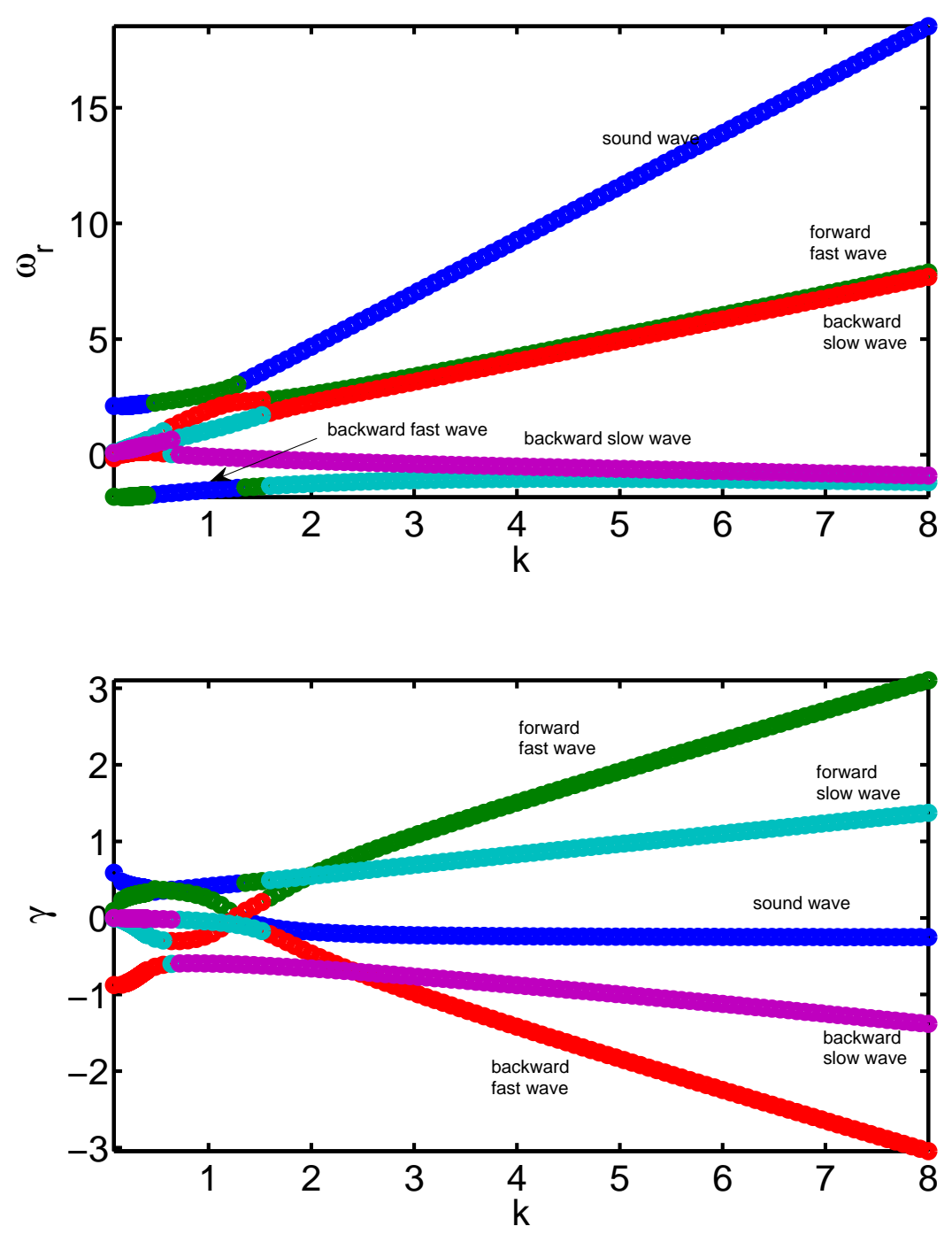

Figure 4. Larger modes $k / k_{c}>1$ (smaller scales) show predominant propagation of forward fast/slow waves as shown in the upper panel. Smaller scales show a decoupling with the sound waves. Lower panel shows unstable modes in the coupled plasma-neutral system for larger modes. Sound waves are found to be stable for the smaller scales.

longer length scale $\left(k / k_{c}<1\right)$ and the scales comparable to the charge exchange length $\left(k / k_{c} \simeq 1\right)$, where $k_{c}=\sqrt{\sigma n_{0}}=2 \pi / l_{c}$ is the charge exchange mode $\left(l_{c}\right.$ charge scale length). Charge exchange interactions not only modify the propagating characteristic of the coupled plasma-neutral modes, but they also alter the growth and damping rates.

We first examine the affect of charge exchange coupling on the longer (than charge exchange length $l_{c}$ ) length scale part of the spectrum. The real part of the complex frequency leads to the sound wave, forward and backward propagating fast/slow magnetosonic waves. This is shown in Fig (2). Alfvénic branch is not shown in 
this figure. Note that the sound waves in the neutral fluid coexist with the plasma modes due to the charge exchange coupling. Furthermore, the sound waves are not destablized by the charge exchange at lower $k$ 's. Interestingly, the charge exchange interactions drive large scales unstable. It is evident from Fig (2), lower panel, that the linearly unstable damping modes coexist with the growing (or unstable) modes. By virtue of this, the mode coupling interactions turn out to be far more complex. An implication of this instability is to lead to an alternate growth and damping of the fast/slow waves. This instability was thought to govern nonlinear modulation of the fast/slow wave in the coupled plasma-neutral system reported in Ref [15] by us. It is further noted that the sound waves begin to decouple from the MHD modes for higher $k>1$ modes. This is an indication that the small scales are not coupled efficiently by the charge exchange interactions. This is further consistent with our nonlinear two dimensional simulations [14] that show a weak coupling between the small scale turbulent fluctuations and charge exchange scales.

We next access a moderately higher wavenumber regime in the spectrum. This regime, depicted in Fig (3), exhibits an entirely different characteristic compared to the one described in Fig (2). Clearly, the forward propagating fast and slow magnetosonic modes dominate over the slow (below zero roots) ones in the higher $k$ spectrum where $k>k_{c}$ (but not $k \gg k_{c}$ ). By contrast, the unstable modes (lower panel in Fig 3) exhibits interesting feature. In that, the linearly unstable fast/slow modes (i.e the second curve from the top) shows initial growth (i.e $\gamma>0$ ) for $k<1.25$. This characteristic behavior reverses for $k>1.25$. Converse is true for the third (from the top) curve. On the other hand, the top and lowest curves corresponding to the forward and backward propagating fast/slow modes show respectively the growth and damping of the instability.

Finally, to investigate the asymptotic behavior of these linearly stable/unstable modes, we extend our analysis to span the higher $k$ modes in the spectrum. This is shown in Fig (4). It becomes clear from this figure that the linear wave propagation (upper panel) show a considerable shift in the frequency towards the forward propagating branch $\left(\omega_{r}>0\right)$, whereas the frequency associated with the backward propagation is found to be reduced considerably. Interestingly, the fast/slow branches associated with the magnetosonic waves appear to have merged for the smaller scales in the $k$-spectrum. The unstable modes, (Fig 4, lower panel), on the other hand exhibit a predictable behavior. It is found that the forward propagating fast/slow modes are driven unstable by the charge exchange coupling in the coupled plasma-neutral system. On the other hand, the backward propagating fast/slow modes are damped by the charge exchange interactions. This is described by the lower panel in Fig (4). It is further observed that the sound waves, in the higher $k$ regime, are decoupled from the predominant MHD modes. This means that charge exchange do not mix up these modes at relatively small characteristic length scales.

\section{Summary and conclusion}

A major outcome from our analysis is that charge exchange interactions in the partially ionized gases, dominated by the coupled plasma-neutral system, in the heliosphere do not directly modify the propagation characteristic of the linear Alfvén waves. This does not necessarily mean that their nonlinear evolution is also unaf-

fected. As a matter of fact, the nonlinear evolution of Alfvén waves is influenced 
by their mode coupling interaction with the fast/slow magnetosonic waves. This is discussed by Shaikh \& Zank (2010). We however find that the charge exchange interaction influence fast/slow mode by linearly driving them unstable.

The linear analysis described in the paper is extremely useful in explaining some of the features of our nonlinear simulations $[14,15]$. The latter show that charge exchange modes modify the helioshperic turbulence cascades dramatically by enhancing nonlinear interaction time-scales on large scales. By contrast, small scale turbulent fluctuations are unaffected by charge exchange modes which evolve like the uncoupled system as the latter becomes less important near the larger $k$ part of the helioshperic turbulent spectrum. This tends to modify the characteristics of helioshperic plasma turbulence which can be significantly different from the Kolmogorov phenomenology of fully developed turbulence.

The onset of modulational instability in Ref [15] is triggered essentially by linear instability process. Our linear stability analysis indicates that the underlying coupled plasmaneutral system possesses fast/slow unstable modes. These modes account for the growing as well as damping of Alfvnic and fast/slow compressive waves. The latter leads to a concurrent damping of the fast/slow compressive mode preceding the growth of Alfvénic mode occurs. When the two modes reach their extremal rise or fall, they reverse their behavior. The fast/slow compressive mode begins to rise at the expense of damping of Alfvénic mode. This process continues nonperiodically and repeats itself in time thereby exhibiting a nearly oscillatory behavior. It then follows that the two modes, i.e. Alfvénic and fast/slow compressive, regulate each other in a predatorprey manner. Dynamically, the Alfvénic mode grows at the expense of fast/slow mode. When Alfvénic mode reaches its maximum amplitude, where the fast/slow mode remains at its lowest magnitude, the latter eats up the Alfvénic mode and vice versa. This process is repetitive.

Our results should find application to a variety of astrophysical environments in which a partially ionized plasma is typical. Examples include the outer heliosheath formed by the interactions of the solar wind with the local interstellar medium, the magnetic collapse of molecular clouds and star formation and the general transfer of energy in partially ionized plasmas surrounding other stellar systems [16].

\section{Acknowledgment}

This work was performed under PH499 capstone course during the summer 2010. The partial support of NASA grants NNX09AB40G, NNX07AH18G, NNG05EC85C, NNX09AG63G, NNX08AJ21G, NNX09AB24G, NNX09AG29G, and NNX09AG62G is acknowledged.

\section{References}

[1] Balsara, D., ApJ., 1996, 465, 775.

[2] Fite, W. L., Smith, A. C. H., and Stebbings, R. F., 1962, Proc. R. Soc. London, em A. 268, 527.

[3] Florinski, V., G. P. Zank, and N. V. Pogorelov (2003), J. Geophys. Res., 108(A6), 1228, doi:10.1029/2002JA009695.

[4] Florinski V., G. P. Zank, N. V. Pogorelov (2005), J. Geophys. Res., 110, A07104, doi:10.1029/2004JA010879. 
[5] Gigliotti, A., Gekelman, W., Pribyl, P., Vincena, S., Karavaev, A., Shao, X., Sharma, A., Papadopoulos, D., 2009, Phys. Plasmas, 16, 092106.

[6] Gekelman, W., 2004, Journal of Geophysical Research, 109, A01311.

[7] Kulsrud, R., and Pearce, 1969, ApJ 156, 445.

[8] Leake, J., Arber, T. D., M. L. Khodachenko, M. L., 2005, Astronomy and Astrophysics, 442, 1091.

[9] Oishi, J. S. and Mac Low, M., 2006, ApJ, 638, 281.

[10] Padoan, P., Zweibel, E., and Nordlund, A., 2000, ApJ, 540, 332.

[11] Pauls, H. L., Zank, G. P., and Williams, L. L., 1995, J. Geophys. Res. A11, 21595.

[12] Shaikh, D., and Zank, G. P., 2008, The Astrophysical Journal, Volume 688, Issue 1, pp. 683-694.

[13] Shaikh, D., and Zank, G. P., 2006, The Astrophysical Journal, Volume 640, Issue 2, pp. L195-L198.

[14] Shaikh, D. and Shukla, P. K., 2009, Physical Review Letters, vol. 102, Issue 4, id. 045004

[15] Shaikh, D., and Zank, G. P., 2006, Physics Letters A 374 (2010) 4538-4542.

[16] Wood, B. E., Harper, G. M.; Muller, H. R., Heerikhuisen, J., Zank, G. P., ApJ, 655, 946, 2007.

[17] Zank, G. P., 1999, Space Sci. Rev., 89, 413-688, 1999. 